\title{
The COPD Assessment Test: what have we learned over its first 5 years?
}

\author{
Paul W. Jones \\ Affiliation: Institute for Infection and Immunity, St George's, University of London, London, UK. \\ Correspondence: P.W. Jones, Institute for Infection and Immunity, St George's, University of London, Cranmer \\ Terrace, London SW17 ORE, UK. E-mail: pjonesdasgul.ac.uk
}

$\boldsymbol{0}$ @ERSpublications What have we learned from the COPD Assessment Test over its first 5 years? http://ow.ly/z8JCd

It is 5 years since the development and first validation of the COPD Assessment Test (CAT) [1], so the review of papers concerning the CAT by GUPTA et al. [2] in this issue of the European Respiratory Journal is very timely. The authors identified over 40 studies using a systematic approach to minimise biases, although they acknowledge that they introduced one bias by selecting papers published only in English, French and Spanish, so eight papers were excluded. That may not be too problematic, as the included papers report data on over 25000 patients from 35 countries across five continents (none from Australasia or Antarctica).

One of the strengths of a systematic review is that it can allow comparison of variability in outcomes across different studies. To this end, the data extracted for this review provide an indication of the reliability of the CAT in different settings, and it is generally very good. For example, the internal consistency measured using Cronbach's alpha was in the range 0.85-0.98 across nine studies. This observation is important because the CAT was developed in English but these studies included Arabic-speaking countries, Brazil, Greece, Japan, South Korea and Turkey. This observation supports the finding by KwON et al. [3], in a direct comparison, that the relationship between the CAT and the St George's Respiratory Questionnaire (SGRQ) was the same across four Asian countries. The review also shows that test-retest reliability is good across studies, with intraclass correlation coefficients ranging $0.80-0.98$, the worst repeatability being in an English language study.

Cross-sectional comparisons with comprehensive disease-specific health status measures such as the SGRQ and Clinical COPD Questionnaire showed the expected good cross-sectional correlations, in the range $0.68-$ 0.92 . Encouragingly, there were also good within-patient longitudinal correlations, with $r$-values of $\sim 0.6$ (longitudinal correlations are always weaker than cross-sectional ones, because the range of within-patient changes is smaller than differences between patients). In contrast, the correlation with modified Medical Research Council (mMRC) grade was much more variable (0.29-0.61), showing that the CAT and the mMRC measure constructs that are rather weakly related. The mean CAT score in mMRC grade 0 patients was 13.8 in the studies reviewed here, showing that the mMRC is relatively insensitive compared with the CAT, at least at the mild end of the spectrum. It also supports the conclusion that, while a mMRC cut point of $\geqslant 1$ identifies a similar proportion of patients to that identified by a CAT cut point of $\geqslant 10$, the make-up these groups is different [4]. This confirms that the implication (made by the Global Initiative for Chronic Obstructive Lung Disease (GOLD) in 2011) that CAT $\geqslant 10$ is equivalent to mMRC $\geqslant 2$ was incorrect, and it supports the GOLD 2014 recommendation that a comprehensive assessment such as the CAT should be the assessment of choice [5].

The review is especially valuable because it provides a useful source of indicative CAT scores for different groups of patients and clinical situations, and the recently published estimate of a minimum clinically important difference of 2 units [6] enables these scores to be put into context. For illustrative purposes,

Received: July 092014 | Accepted: July 092014

Conflict of interest: None declared.

Copyright (C)ER 2014 
I have calculated the simple mean of CAT scores across the published studies reviewed here and expressed them as integer values, because CAT scores contain no decimals at an individual-patient level. They are purely indicative and the full details are clearly presented in the review, but they may provide a useful broad guide. In healthy subjects, the mean score is 7 . When classified by GOLD grade, the CAT scores are: grade I, 14; grade II, 16; grade III, 20; and grade IV, 23. It is clear that even mild airflow limitation in patients diagnosed with COPD is associated with CAT scores that indicate "moderate impact" of COPD on their health (www.catestonline.org). In contrast, people recruited from the workplace may have CAT scores $<10$, despite meeting spirometric criteria for chronic obstructive pulmonary disease (COPD) [7].

It is encouraging to see the number of prospective studies identified. They show that the CAT is responsive to pulmonary rehabilitation ( $\sim 3$ units, on average), which is comparable to changes seen with the Chronic Respiratory Questionnaire and SGRQ [8]. They also show that the difference between stable and exacerbating patients is consistently large, typically 5 units, with an average improvement on recovery of 7 units. One important group of studies is still missing: the effect of pharmacological treatment. Another area where evidence is limited is predictive validity, although a recent study, too late for this review, has shown that CAT scores predict exacerbations even in COPD patients already identified as being at high risk, based on their history [9].

There are also other gaps in the literature. For example, more data are needed in patients $<50$ and $>70$ years of age, and in patients with severe limitation in activity due to breathlessness, such as those with mMRC grade 4 whose scores are little different from those in mMRC grade 3 . There are also currently very few data about long-term trends in CAT scores. For example, what is the average rate of worsening over time? Are there "fast" and "slow" decliners? What role does the CAT have when combined in comprehensive assessment? For example, a recent analysis suggested that worsening COPD, as assessed using GOLD 2011, appears to be associated with increased risk of metabolic syndrome [10]. This application could usefully be explored more, as could the application of the CAT as a case-finding tool, not to identify people with airflow limitation, but to find symptomatic undiagnosed patients with COPD who may benefit from treatment. The data presented in this review suggest that a cut point of 10 might be a starting point for such a study.

The original idea behind the CAT's development was that it should aid communication between patient and clinician [11]. Use of modern psychometrics in its development allows it to do much more than that. This review has shown that it provides reliable measurement of a complex construct, overall health status impairment, using a very small number of items. CAT scores can aid assessment of patients and communication between healthcare professionals in routine practice, and help characterise patients and measure outcomes in clinical studies. The fact that this review of the CAT identified so many studies, performed in so many patients, in such a short time is clearly a testament to its strengths: it is short, easy, reliable, responsive and very cheap to use.

\section{References}

1 Jones PW, Harding G, Berry P, et al. Development and first validation of the COPD Assessment Test. Eur Respir J 2009; 34: 648-654.

Gupta N, Pinto LM, Morogan A, et al. The COPD assessment test: a systematic review. Eur Respir J 2014; 44: 873-884. Kwon N, Amin M, Hui DS, et al. Validity of the Chronic Obstructive Pulmonary Disease Assessment Test (CAT) translated into local languages for Asian patients. Chest 2013; 143: 703-710.

4 Jones PW, Adamek L, Nadeau G, et al. Comparisons of health status scores with MRC grades in COPD: implications for the GOLD 2011 classification. Eur Respir J 2013; 42: 647-654.

5 Global Initiative for Chronic Obstructive Lung Disease. Global Strategy for the Diagnosis, Management and Prevention of Chronic Obstructive Pulmonary Disease. www.goldcopd.org/uploads/users/files/GOLD_Report_ 2014_Jun11.pdf

6 Kon SSC, Canavan JL, Jones SE, et al. Minimum clinically important difference for the COPD Assessment Test: a prospective analysis. Lancet Respir Med 2014; 2: 195-203.

7 Nishimura K, Mitsuma S, Kobayashi A, et al. COPD and disease-specific health status in a working population. Respir Res 2013; 14: 61.

8 Lacasse Y, Goldstein R, Lasserson TJ, et al. Pulmonary rehabilitation for chronic obstructive pulmonary disease. Cochrane Database Syst Rev 2006; 4: CD003793.

9 Lee SD, Huang MS, Kang J, et al. The COPD Assessment Test (CAT) assists prediction of COPD exacerbations in high-risk patients. Respir Med 2014; 108: 600-608.

10 Jones PW, Nadeau G, Small M, et al. Characteristics of a COPD population categorised using the GOLD framework by health status and exacerbations. Respir Med 2014; 108: 129-135.

11 Jones P, Harding G, Wiklund I, et al. Improving the process and outcome of care in COPD: development of a standardised assessment tool. Prim Care Respir J 2009; 18: 208-215. 